\title{
Pulsating Flow and Platelet Aggregation
}

\author{
Xin-She Yang \\ Department of Engineering, University of Cambridge, \\ Trumpington Street, Cambridge CB2 1PZ, UK \\ xy227@eng.cam.ac.uk
}

\begin{abstract}
Platelet aggregation has been modelled as a nonlinear system of viscous flow and pulsating flow based on the Fogelson's continuum model. By solving nonlinear governing equations and the coupling between flow and platelet aggregates in the cylindrical coordinates using the finite element method, we can see how the platelet aggregation forms under the pulsating flow conditions. Numerical simulations show that the aggregates are stretched by the flow in the dynamic equilibrium of the link forces and background flow. In addition, significant elastic stress can be developed to maintain the shape and integrity of the platelet aggregates.
\end{abstract}

\section{Introduction}

Platelet aggregations are important in physiological processes such as thrombus and haemostasis. However, these processes are very complicated and many factors and activities occur at the same. For example, physiological response in haemostasis to blood vessel injury involves the cell-cell adhesive platelet aggregation and coagulation. Platelets are tiny oval structure with diameter of 2-4 micrometers. They are active in blood with a half-life of 8-12 days. Non-active platelets flow free in blood vessels in a dormant state. Activating chemicals such as ADP initiated by injury can induce platelet aggregation in the blood plasma. A platelet's surface membrane can be altered so that the platelet becomes sticky, and thus capable of adhering to other activated platelets and the vessel walls. Although platelets only consist of about $0.3 \%$ in concentration and yet occur in enormous numbers about 250 millions per millilitre $[2,3,6]$. Once triggered by the chemicals, the active platelets start to clot so as to restrict the blood flow at the injury site. To prevent uncontrolled overspreading of activated platelets, a chemical inhibitor, thrombin, is also involved.

There have been extensive studies on the biological and physiological effect of platelet aggregations. Some mathematical models and computational models are very instructive for the modelling of the detailed mechanism of the formation of platelet aggregates [3-6]. Most of these studies use the Navier-Stokes equations with simplified boundary conditions or the steady-state approximation. However, the real blood vessel system has complex geometries due to branching and atherosclerotic plague deposition inside the vessels. In addition, the flow velocity and pressure are even more complicated by the pulsating process from the heart pumping process. Thus, the more realistic modelling shall include the pulsating effect on the flow and 
platelet aggregations. In this paper, we intend to extend the existing models to include the pulsating flow and the platelet aggregations in the corresponding environment.

\section{Fogelson's Model}

Fogelson first formulated a continuum model for platelet aggregation process [3]. The full model consists of a system of coupled nonlinear partial differential equations. We use the simplified version of Fogelson's model with the following equations:

$$
\begin{aligned}
& \nabla \cdot \boldsymbol{u}=\mathbf{0}, \\
& \rho\left(\boldsymbol{u}_{\boldsymbol{t}}+\boldsymbol{u} \cdot \nabla \boldsymbol{u}\right)=-\nabla p+\mu \nabla^{2} u+f+\beta \nabla \sigma, \\
& \boldsymbol{\sigma}_{\boldsymbol{t}}+\boldsymbol{u} \nabla \boldsymbol{\sigma}=\boldsymbol{\sigma} \nabla \boldsymbol{u}+(p \nabla \boldsymbol{u})^{\boldsymbol{T}}, \\
& \phi_{t}+\boldsymbol{u} \cdot \nabla \phi=r,
\end{aligned}
$$

where the first two equations are the Navier-Stokes equations for incompressible fluid flow $\boldsymbol{u}=(U, V, W)$ and $p$ pressure. The coefficient $\beta$ is constant and $\boldsymbol{f}$ is the force density. The last term is due to the cohesion of platelets. The third equation is for the cohesion-stress tensor $\sigma$. The last equation is for the concentration $\phi$ of the active platelets and the production rate $r$ can be considered as a function of concentrations of platelets and the activating chemicals. The governing equations are nonlinear and the flow is coupled with the formation of platelet aggregates. Thus the full solution necessitates efficient numerical methods. As the blood flow is slow and viscous, so the first two equations can be simplified for the case of viscous flow and the zero force $f$. We have

$$
\nabla \cdot \boldsymbol{u}=0, \quad \rho \boldsymbol{u}_{t}=\mu \nabla^{2} \boldsymbol{u}-\nabla p+\beta \nabla \sigma
$$

In most cases, the blood flow is one-dimensional tubular flow, it is convenient to choose the local coordinates so that the $z$-axis is in the direction of the local blood flow. In this case, we can assume the pulsating pressure (far field) in the form

$$
p_{z}=\partial p / \partial z=A \cos (\omega t)+\gamma
$$

where $A$ is a constant. In fact, we can assume any known function form for the pressure gradient in terms of a Fourier expansion

$$
p_{z}=g(t)=\gamma+\sum_{n=1}^{N}\left[A_{n} \cos \left(\omega_{n} t\right)+B_{n} \cos \left(\omega_{n} t\right)\right] .
$$

\section{Simulation and Results}

Although the equations are coupled, they can be solved using the well-developed finite element method together with the Newton-Raphson iteration procedure for the 
nonlinearity. By solving the problem in the cylindrical coordinates, we can see how the platelet aggregation forms and develops under the pulsating flow conditions.

\subsection{Shear Stress Distribution and Variation}

To simulate the tubular flow and the stress development, we first study the flow at the bifurcation region where one large vessel is branched into two smaller vessels. For a given pulse, one can calculate the wall shear stress and stress variation with time after the peak R-wave of the given pulse. Figure 1 shows the shear stress distribution and the mean wall shear stress variation. The major feature of the computed shear stress variation is consistent with the experimental results. It is worth pointing out that there exists a peak for wall shear stress for a given pulsating pressure gradient, and thus the peak shear stress could directly affect the rupture if the stenosis or plague is presented at the region concerned. The shear stress is higher at bifurcation regions and the stress level can reach as high as $15 \mathrm{~Pa}$ for a blood vessel with a diameter of $1 \mathrm{~mm}$. As the flow is pulsating, the shear stress and pressure vary with time. The mean shear stress at wall varies significantly after the R-wave starts. It first increases quickly to a peak at $\mathrm{t}=0.065$ and then decreases exponentially with time.
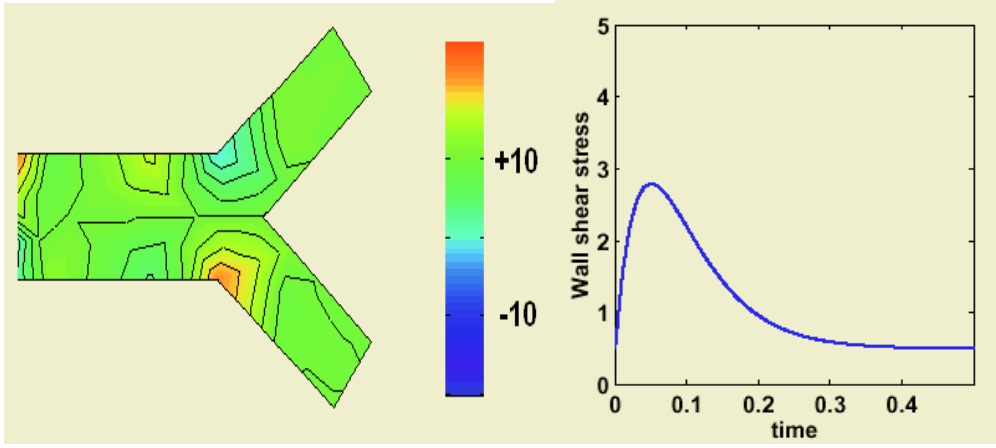

Fig. 1. Shear stress distribution at the bifurcation region and wall stress variation with time

\subsection{Platelet Aggregation}

In the case of pulsating flow in a cylindrical vessel with a diameter of $1 \mathrm{~mm}$, a small injury (point A) with a size of less than $0.1 \mathrm{~mm}$ occurs and thus releases the ADP chemical to activate the platelet aggregation, so platelets starts to deposit at the injury site, and the size of the aggregation starts to increase. Figure 2 show the platelet aggregation at the different times $t=1,10,50$ seconds after the injury. The flow is from left to the right with the initial constant flow field. The boundary condition for the pressure in the far field is the pulsating function given earlier in equation (7). For simplicity, we have used the zero reaction rate $(r=0), \gamma=0, \beta=1$, and the normalized concentration so that $\phi=0$ for no platelets and $\phi=0.5$ for solid platelet aggregates.

We can see clearly that the concentration of activated platelets is much higher at the injury site than in the blood. The aggregation rate is proportional to the 


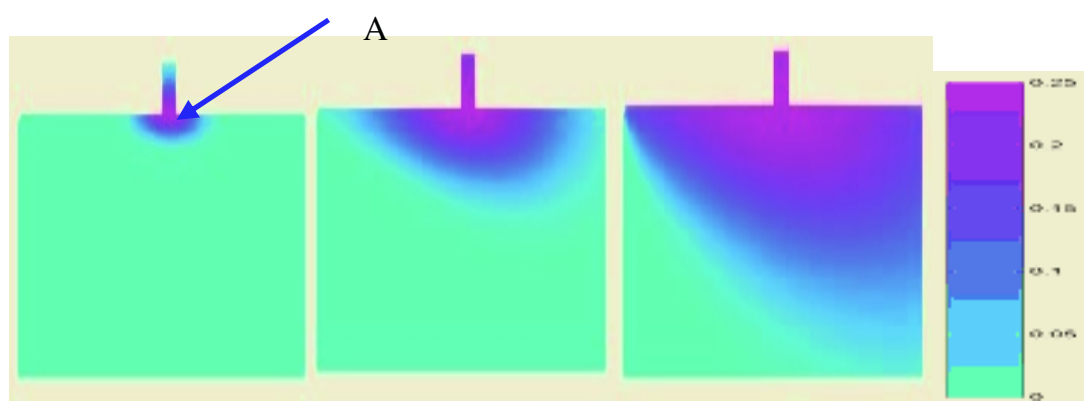

Fig. 2. Formation of platelet aggregation at different times $(t=1,5,50)$

concentration of the activated platelets, and thus the size and shape of the platelet aggregation can be represented in terms of the concentration as shown in the figure. The aggregates are stretched by the flow in the dynamic equilibrium of the link forces and background flow. As the platelets aggregate, the injured vessel will be blocked severely after some time, and it may take 100 seconds to reach this stage.

\section{Conclusions}

We have simulated the platelet aggregation by using the Fogelson's continuum model with nonlinear governing equations and the coupling between flow and platelet aggregates. By solving the problem in the cylindrical coordinates using the finite element method, we can see how the platelet aggregation forms under the pulsating flow conditions. Numerical simulations show that the aggregates are stretched by the flow in the dynamic equilibrium of the link forces and background flow. In addition, significant elastic stress can be developed to maintain the shape and integrity of the platelet aggregates.

\section{References}

1. Chakravarty, S., Mandal P. K.: Mathematical modelling of blood flow through an overlapping arterial stenosis. Math. Comput. Modelling, 19 (1994) 59-70.

2. David, T., Thomas, S., Walker, P. G.: Platelet deposition in stagnation poiint flow: an analytical and computional simulation. Medical Engineering and Physics, 23 (2001) 229-312.

3. Fogelson, A.: Continuum models of platelet aggregation: formulation and mechanical properties. SIAM J. Appl. Math., 52 (1992) 1089-1110.

4. Gurevich, K. G., Chekalina, N. D., Zakharenko, O. M.: Application of mathematical modelling to analysis of nerves growth factor interaction with platelets. Bioorganic Chemistry, 27(2000)57-61.

5. Guy, R. D., Fogelson, A. L.: Probabilistic modelling of platlet aggregation: effects of activation time and receptor occupancy. J. Theor. Biol., 219(2002) 33-53.

6. Keener, J. and Sneyd, J.: Mathematical Physiology, Springer-Verlag, Berlin Heidelberg New York (1998).

7. Zienkiewicz, O. C. and Taylor, R. L.: The Finite Element Method, Vol. I/II, McGraw-Hill, 4th Edition, (1991). 